\title{
COVID-19-Associated Acute Respiratory Distress Syndrome: Lessons from Tissues and
} Cells

\author{
Elizabeth A. Middleton, MD, Guy A. Zimmerman, MD*
}

\author{
KEYWORDS \\ - COVID-19 • SARS-CoV-2 • ARDS • Acute lung injury • Histopathology \\ - Vasculopathy
}

\section{KEY POINTS}

- Specific histologic patterns with pathogenetic implications are emerging from clinical and experimental studies of coronavirus disease 2019 (COVID-19) acute lung injury.

- Physiologic variables and clinical outcomes in COVID-19-associated acute respiratory distress syndrome linked to histopathologic patterns may be identified.

- These studies may provide a rationale for targeted interventions based on specific cellular events and temporal phases of COVID-19 acute lung injury.

\section{INTRODUCTION}

Patients with coronavirus disease 2019 (COVID-19) induced by severe acute respiratory syndrome coronavirus 2 (SARS-CoV-2) frequently develop acute, precipitous respiratory failure with features of the acute respiratory distress syndrome (ARDS). ${ }^{1}$ Understanding the biology, mechanisms of acute lung injury, and pathophysiology of COVID-19-associated ARDS is essential for its rational management, ${ }^{1,2}$ but these elements are largely uncharacterized. ARDS is a common, complex, and lethal syndrome that is caused by a spectrum of infectious and noninfectious insults. ${ }^{3,4}$ COVID-19-associated ARDS may be, in large part, similar to ARDS of other causes. ${ }^{5,6}$ Alternatively, it may have novel features and it is possible, and perhaps likely, that COVID-19-associated ARDS represents a unique phenotype..$^{7,8}$ It is also possible that there is biological and physiologic heterogeneity and that there are subphenotypes of COVID-19-associated ARDS, as there are in classic ARDS (a term that is used here to indicate ARDS unrelated to COVID-19). ${ }^{2,4,7}$

Division of Pulmonary and Critical Care Medicine, Department of Internal Medicine, Program in Molecular Medicine, University of Utah School of Medicine, Eccles Institute of Human Genetics, 15 North 2030 East, Room \#4220, Salt Lake City, UT 84112, USA

* Corresponding author.

E-mail address: guy.zimmerman@u2m2.utah.edu 
Histopathology and clinical cell biology are fundamental to understanding human diseases and for elucidation of their mechanisms and physiologic consequences, and may be critical for precise characterization of new or emerging syndromes. For clinicians, description of the anatomy of the disorder provides correlates for interpretation of imaging and other diagnostic measures, understanding pathophysiology, and formulating therapeutic strategies; for translational investigators, human histopathology is a basis for devising reduced experimental models and for evaluating outcomes in surrogate in vivo experiments; for medical scientists new to a field, histopathology can provide an unbiased sense of complexity of a disease and insights regarding the cellular and molecular issues that underpin it. Linking pathologic patterns to clinical variables can be particularly informative. ${ }^{9}$ This article profiles available information on the pathology of SARS-CoV-2 pneumonia, focusing on histology and cellular characterization and lessons and questions that these studies provide regarding COVID19-associated ARDS.

\section{OVERVIEW OF THE PATHOLOGY OF SEVERE ACUTE RESPIRATORY SYNDROME CORONAVIRUS-2 PNEUMONIA}

Current synthesis of the pathology of SARS-CoV-2 pneumonia is based on reports of autopsies, more limited postmortem sampling, surgically excised lung tissue, and cytologic analysis. Multiple studies of documented SARS-CoV-2 infection from Asia, Europe, and the United States have appeared. Early cases and case series are summarized in reviews. ${ }^{10-13}$ This article also discusses findings reported in selected early and more recent primary reports.

Macroscopic features of the lung in COVID-19 are nonspecific and include edema, hemorrhage, and thrombosis. ${ }^{12}$ Lung weights are substantially increased. In an international report of 68 autopsies, the combined lung weight was greater than $1300 \mathrm{~g}$ (normal average $840 \mathrm{~g}$ ) in $92 \%$ of cases. ${ }^{14}$ In a smaller series, the mean weight of lungs from subjects with COVID-19 $(1681 \pm 49 \mathrm{~g})$ was greater than that of uninfected control lungs $(1045 \pm 91 \mathrm{~g}$ ) but lower than that of patients dying of influenza-associated ARDS $(2404 \pm 560 \mathrm{~g}){ }^{15}$ Macroscopic involvement is frequently patchy but there can be extensive consolidation, corresponding with a spectrum of patterns on diagnostic imaging. ${ }^{11,12,16}$

In early case reports and series, the most commonly reported histologic finding, by far, was diffuse alveolar damage (DAD), ${ }^{10-13}$ including cellular features of the acute, exudative, and proliferative, or organizing, phases (Box 1, Fig. 1). More recent autopsy series, some multicenter involving large numbers of patients, extend early findings and frequently also emphasize pulmonary vascular involvement and apparent temporal evolution of acute lung injury. ${ }^{14,15,22-32}$ Tracheobronchial injury and inflammation, independent of intubation and mechanical ventilation, have been documented in addition to alveolar involvement. ${ }^{14,30}$ The conclusion is that SARS-CoV-2 pneumonia is a complex respiratory disorder involving the tracheobronchial, alveolar, and vascular compartments. ${ }^{11,12,14}$

Evidence for viral infection of tracheobronchial and alveolar epithelial cells (AECs) by ultrastructure, immunohistochemistry, or in situ hybridization has been a consistent finding in COVID-19; detection of viral particles or markers in endothelial and immune cells has been reported in some, but not all, studies. ${ }^{11-15,22,23,28,30,32-35}$ Angiotensinconverting enzyme 2 (ACE2), a requisite component of the molecular system by which SARS-CoV-2 enters host cells, was detected on alveolar epithelial and endothelial cells. ${ }^{15}$ Persistent viral infection may drive ongoing focal alveolar injury and clinical manifestations ${ }^{14,23}$ but is absent in the organizing phase of DAD in some patients. ${ }^{32}$ 


\section{Box 1}

Diffuse alveolar damage is the characteristic histologic pattern of classic acute respiratory distress syndrome

DAD was originally characterized as a nonspecific response to acute or subacute alveolar injury incited by a variety of insults, alone or in combination. Specific histologic features identifying early exudative and later proliferative phases, often progressing to extensive interstitial fibrosis, were described ${ }^{17}$ (see Fig. 1). Ultrastructural analysis confirmed and extended observations by light microscopy. ${ }^{18,19}$ Hyaline membranes, alveolar epithelial cell (AEC) type I (pneumocyte) injury and loss, interstitial and alveolar edema, and interstitial and alveolar inflammatory infiltrates are key histologic features of the acute exudative phase. The inflammatory infiltrate was originally described to be mononuclear, ${ }^{17}$ but neutrophils are prominent in classic DAD of many causes. ${ }^{4,20}$ Fibrin thrombi are common in alveolar capillaries and pulmonary arterioles. Platelets are frequently detected in microvascular thrombi, especially when specific markers are used. ${ }^{17-19,21}$ Intra-alveolar fibrin, hemorrhage, and cellular debris are variably seen. Ultrastructural studies reveal denuded alveolar epithelial basement membrane and cellular details of epithelial and endothelial injury. ${ }^{18,19}$ The organizing, proliferative phase is indicated by AEC II (type II pneumocyte) hyperplasia and by interstitial fibrosis that can be severe. ${ }^{17-19}$ At present, hyaline membranes are considered the required feature for pathologic diagnosis of DAD in classic ARDS, and the other elements of the histologic pattern are used to establish its evolutionary stage. ${ }^{21}$

Histologic patterns that vary from the dominant DAD phenotype have been reported, ${ }^{10,11,14,22,25,33-35}$ including acute fibrinous and organizing pneumonia. ${ }^{10,36}$ They may be sentinel subsets that indicate biological heterogeneity, potentially underlying clinical heterogeneity. ${ }^{2}$

In addition to the respiratory system, multiple studies have examined other organs and tissues. Although this article does not review extrapulmonary features of SARSCoV-2 infection, they indicate, along with clinical manifestations and circulating biomarkers, that SARS-CoV-2 infection is frequently a systemic syndrome. ${ }^{1,10-13,16}$

There are several caveats regarding current reports of the pathology of COVID-19. The lung tissue examined to date has largely been from elderly patients, frequently with a variety of comorbidities, consistent with the well-known susceptibility to severe SARS-CoV-2 infection and increased mortality in this population. Common features of the lung pathology could therefore partly be caused by responses of the aged, multiply compromised lung. Nevertheless, observations in nonhuman primates indicate that DAD occurs across the age spectrum and in otherwise healthy lungs in response to SARS-CoV-2 infection (discussed later). A second issue is that most of the lung tissue from patients with COVID-19 examined so far is from patients that expired well after onset of the clinical illness, often after days or weeks of medical intervention and intensive care unit support. Thus, the early patterns of lung involvement in SARS-CoV-2 infection in humans are not known. Tissue from patients undergoing lung resection while in the undiagnosed, apparently early, phase of COVID-19 infection provides useful but limited information. ${ }^{37-39}$ Experimental animal models ${ }^{40}$ will likely be essential in addressing this unknown feature. Experimental animal studies will also likely be the only avenue immediately available for determining patterns of histologic and cellular evolution in SARS-CoV-2-induced acute lung injury, although molecular imaging or histology-specific biomarkers ${ }^{21,31}$ may be informative in humans in the future. In addition, virtually no patients in reports of COVID-19 lung histopathology have been ascertained to have ARDS according to the Berlin definition ${ }^{3,4,9}$ or other consensus criteria, although relevant clinical and physiologic data are available for some. Therefore, it is currently necessary to extrapolate existing histopathologic findings to COVID-19associated ARDS. 


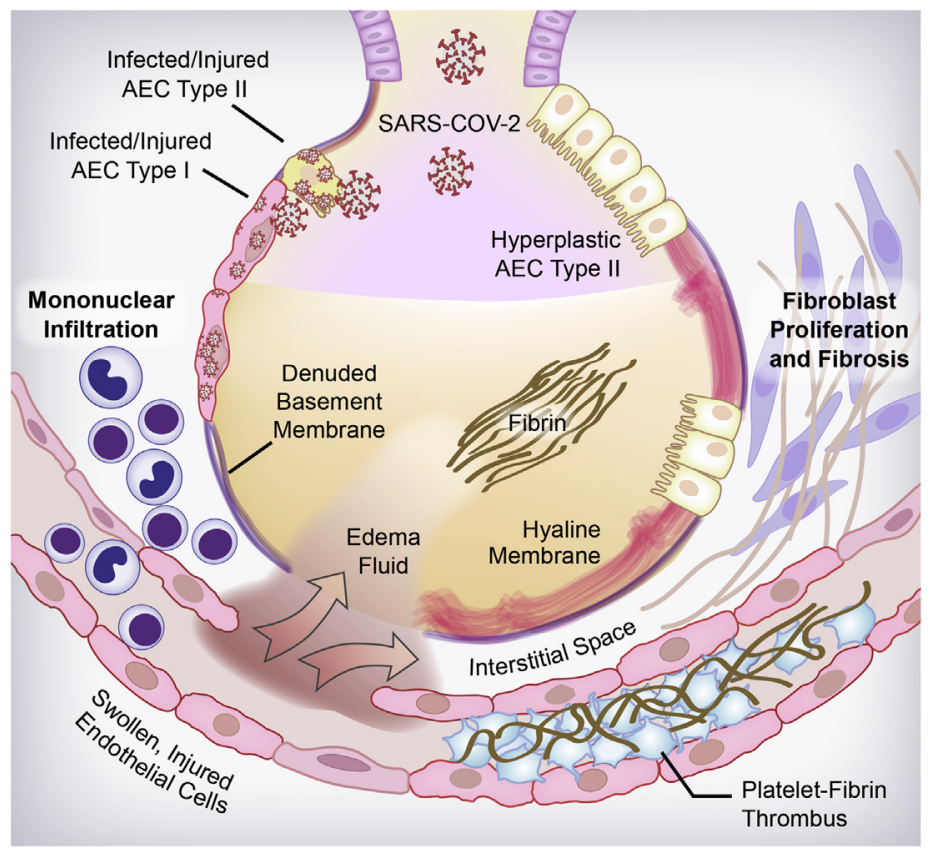

Fig. 1. DAD is the histologic signature of COVID-19 acute lung injury. The pattern and cardinal features of DAD (see Box 1) have been consistently observed in the lungs of patients with documented SARS-CoV-2 infection, including in autopsies of critically ill subjects described to have COVID-19-associated ARDS. Frequently hyaline membranes and alveolar epithelial cell (AEC) type II hyperplasia occur in the same foci of affected lung, indicating temporal overlap and potentially evolution of acute lung injury. Other histologic features besides the cardinal criteria of DAD, including squamous metaplasia, AEC atypia, and intense scarring with obliteration of alveolar spaces, have been variably reported. Variations in the cellular makeup of the inflammatory infiltrate, including the specific pattern of acute neutrophilic bronchopneumonia together with DAD and additional variant histologic patterns, have also been reported. See text for details. DAD was noted to be caused by viral pneumonias in the classic description (Katzenstein and colleagues ${ }^{17}$ ) and is the characteristic histologic pattern in the lungs in SARS, Middle East respiratory syndrome, and pandemic influenza.

\section{DIFFUSE ALVEOLAR DAMAGE: THE SIGNATURE BUT NONSPECIFIC HISTOLOGIC PATTERN OF CLASSIC ACUTE RESPIRATORY DISTRESS SYNDROME AND CORONAVIRUS DISEASE 2019 LUNG INJURY}

DAD is a specific constellation of histologic features that defines a characteristic but nonspecific pattern of response to acute or subacute lung injury ${ }^{17}$ (see Box 1, Fig. 1). It is the signature pathologic lesion and is central in current concepts of ARDS, although not all patients with classic ARDS have DAD on autopsy or diagnostic lung biopsy examination and the physiologic syndrome of ARDS can be associated with other histologic patterns. . $^{3,9,21}$ The presence of hyaline membranes is a requisite criterion for DAD diagnosis, and other histologic features are used to determine evolutionary stages of the response. ${ }^{21}$

As noted previously, DAD is a consistent, almost ubiquitous, finding when lungs from decedents with COVID-19 are examined. ${ }^{10-15,22-32,35}$ DAD is also a key 
component of the acute lung injury induced by coronaviruses in Middle East respiratory syndrome (MERS-CoV) and severe acute respiratory syndrome (SARS-CoV) infection. ${ }^{11,12,24}$

DAD in lungs of patients infected with SARS-CoV-2 could not be differentiated from DAD in acute lung injury induced by other insults when early case reports and case series were reviewed. ${ }^{12} \mathrm{~A}$ focused study concluded that DAD in COVID-19 infection is morphologically indistinguishable from DAD of other causes. ${ }^{29}$ This determination was based on consensus blinded analysis of histopathology of lungs from hospitalized patients with SARS-CoV-2 infection, patients infected with SARS-CoV-2 who died in the community, and historical hospitalized and outpatient controls previously determined to have DAD induced by sepsis, lung infection, or other conditions, including hospitalized controls assessed to have clinical ARDS. The findings were interpreted as indicating that DAD (without distinctive histologic features that differentiate it from DAD of other causes) is the primary manifestation of COVID-19-associated lung injury in patients who die in the hospital or in the community. A corollary was that mechanical ventilation and high inspired oxygen concentration are not primary drivers of the histologic changes. ${ }^{29}$ Both supportive interventions induce the DAD phenotype..$^{3,17}$

Correlation of the histologic pattern of DAD with physiologic data and clinical variables in classic ARDS has been informative. ${ }^{9,21,41,42}$ Evolution of histologic features of DAD in patients with classic ARDS suggests a time-dependent set of biological events that alter physiologic variables and might be modified by time-sensitive interventions. ${ }^{41}$ Cellular features indicating evolution of acute lung injury seem to also correlate with duration of disease in COVID-19. ${ }^{14,22,28,32}$ A recent histologic and transcriptomic analysis extending findings in one of the early major COVID-19 autopsy series suggests distinct phases of immune disorder, varying in interferon-stimulated genes, cytokines, viral load, and cellular injury, and natural progression culminating in DAD. ${ }^{31}$ If verified, this may provide a basis for timing of administration of targeted therapeutics in clinical trials and, ultimately, in practice. Evaluation of the impact of DAD on physiologic variables and response to supportive measures in COVID-19-associated ARDS $^{5,6}$ requires future study.

Examination of the complex relationship between DAD and classic ARDS indicates that the presence of DAD influences clinical outcomes. ${ }^{21}$ ARDS with DAD detected by open lung biopsy is associated with higher mortality than is ARDS in the absence of DAD. ${ }^{43}$ This finding may also be true of COVID-19-associated ARDS, but it is unclear whether lung biopsy will be widely used in COVID-19 because of risk of viral dissemination and other concerns, underscoring the potential benefits of biomarkers and molecular imaging techniques that reflect this histologic pattern. ${ }^{21,31}$

Although DAD in COVID-19 and DAD in acute lung injury of other causes are indistinguishable by light microscopy, ${ }^{12,29}$ they may be different at the molecular level. Future analysis of clinical lung tissue and bronchoalveolar lavage (BAL) samples, and samples from in vivo and in vitro experimental models, may reveal unique biochemical and cellular features that influence evolution of the alveolar injury or its resolution and repair. ${ }^{4}$

\section{ALVEOLAR IMMUNE EFFECTOR CELLS IN CORONAVIRUS DISEASE 2019- ASSOCIATED LUNG INJURY}

Leukocytes are a common feature of DAD (see Box 1), classically as a prominent interstitial infiltrate. ${ }^{17}$ Inflammatory cells also concentrate in microvessels in numbers greater than those seen in the normal lung, suggesting intravascular activation and sequestration, and accumulate in the alveolar spaces in some cases. ${ }^{4,18,19}$ 
In COVID-19-associated DAD, perivascular and interstitial mononuclear cell infiltrates of variable intensity composed primarily of cluster of differentiation (CD) $4+$ and CD8+ lymphocytes were commonly reported in early studies and were assessed to be consistent with viral infection. ${ }^{10-13}$ Macrophages may have particular activities that drive acute lung injury early in the evolution of SARS-CoV-2 infection. ${ }^{31,37-39,44,45}$ Limited early reports of BAL sampling also indicated predominant lymphocytic and mononuclear cell inflammation, including plasma cells and macrophages. ${ }^{46-48}$ Although characteristic of viral infection, mononuclear leukocyte infiltrates are also found in acute lung injury of other causes, including oxygen toxicity ${ }^{17}$ and malariaassociated ARDS. ${ }^{49}$

Angiocentric accumulation of lymphocytes in the alveolar perivascular interstitium and microvessels was observed in lung tissue from patients with COVID-19 15 and SARS-CoV-2-infected primates. ${ }^{45}$ CD68+ macrophages and an activated T-cell signature correlated with DAD in a detailed study of lungs from COVID-19 decedents involving histology, immunophenotyping, and transcriptomics. ${ }^{31}$ The activities and mechanisms of accumulation of alveolar lymphocytes in COVID-19 are unknown; they are suggested to contribute to both viral clearance and acute lung injury based on histologic and blood analysis. ${ }^{31,50}$ In influenza infection, signaling interplay between lymphocyte subsets, injured alveolar cells, and fibroblasts influences immune cell accumulation and lung dysfunction, suggesting that similar events may occur in COVID-19. ${ }^{51}$ Circulating platelet-lymphocyte and platelet-monocyte aggregates were increased in the blood of patients with COVID-19 compared with samples from control patients, ${ }^{52,53}$ indicating one potential mechanism of lung accumulation of mononuclear leukocytes and showing interaction of key immune effector cells. ${ }^{54}$

Myeloid leukocyte subsets may also have critical effector activities in COVID-19 pneumonia. ${ }^{44,47,55}$ Neutrophils (polymorphonuclear leukocytes) are hallmarks of classic ARDS induced by a variety of common infectious and noninfectious triggers, ${ }^{20}$ but their involvement in SARS-CoV-2 lung infection is unclear. Neutrophils were infrequently identified in early COVID-19 autopsies, surgical cases, and BAL samples. ${ }^{12,38,39,46,48}$ They were much less frequently detected by quantitative scoring in alveoli of patients with COVID-19 than in the lungs of patients dying of influenza. ${ }^{15}$ Nevertheless, neutrophils were present in lung tissue from some patients with COVID-19. ${ }^{10,12,14,22,33-37,56}$ One possibility is that neutrophils in these samples were indicators of superimposed bacterial pneumonia, and it was suggested that they are not effector cells in the primary inflammatory response to SARS-CoV-2 because they are not typically found in the lung in uncomplicated viral infection. ${ }^{12}$ Autopsy evidence shows that bacterial pneumonia occurs in COVID-19 infection (discussed later), supporting this possibility.

Alternative possibilities are that neutrophils are transient cellular components or are a subpopulation of effector cells in SARS-CoV-2 lung infection, or that DAD with neutrophilic inflammation is a distinct phenotypic response to SARS-CoV-2. Observations in nonhuman primates suggest that SARS-CoV-2 has the biological potential to directly or indirectly trigger early alveolar accumulation of neutrophils in combination with mononuclear leukocytes. Neutrophils were present in cellular infiltrates together with lymphocytes and macrophages in foci of inflammation localized with AEC type I and AEC II positive for SARS-CoV-2 antigen in necropsy samples collected 4 days after infection of cynomolgus macaques. ${ }^{57}$ Neutrophils were also identified together with mononuclear leukocytes in focal areas in lungs of rhesus macaques sacrificed 2 to 4 days after infection with SARS-CoV-2. ${ }^{58,59}$ These models further showed key features of DAD at these early time points after infection. Nevertheless, evolution and changes in the immune effector cell populations and, specifically, persistence of neutrophils were not determined, which is an issue for future investigation. 
One potential consequence of neutrophil accumulation in inflammatory syndromes is generation of neutrophil extracellular traps (NETs). NETs have both defensive and injurious activities, are triggered by viral and nonviral pathogens, and are generated in ARDS. ${ }^{49,60}$ Neutrophil accumulation in capillaries, alveoli, and airways of 3 patients dying of COVID-19 suggested that NETs contribute to the pathobiology of SARS-CoV2 infection. ${ }^{60}$ Neutrophils in the process of NET formation (NETosis) and extracellular NET lattices were subsequently shown in autopsy sections of lung tissue from decedents COVID-19, 1 of whom expired after 5 days of intensive care treatment of ARDS (with concomitant empiric treatment of community-acquired pneumonia) and 2 others who died shortly after emergency room admission. ${ }^{61}$ Plugs composed of neutrophils alone, and neutrophils interpreted to be undergoing NETosis based on swollen nuclei that stained positively for citrullinated histone $\mathrm{H}_{3}$, on a background of DAD with interstitial T-lymphocyte infiltrates, were observed in lungs of a subset of a second series of patients dying of COVID-19; 9 out of 21 subjects (43\%) had fungal or bacterial superinfections in this report. ${ }^{35}$

In correlative studies, myeloperoxidase/DNA complexes, a plasma marker of NETs, were increased in blood samples from hospitalized patients with COVID-19, including subjects with ARDS. ${ }^{61}$ The levels were directly correlated with severity of illness and inversely correlated with oxygenation indexed by the $\mathrm{PaO}_{2} /$ fraction of inspired oxygen ratio. In parallel, neutrophils from patients with COVID-19 underwent unstimulated NETosis in vitro and plasma from patients with COVID-19 triggered NET formation by control neutrophils, a process that could be blocked by a novel inhibitor. ${ }^{61}$ In a different analysis of circulating blood cells and postmortem lung tissue, aberrant patterns of neutrophil and platelet activation and plateletneutrophil aggregate formation were related to severity of COVID-19 pneumonia, providing evidence for immunothrombosis involving platelets, neutrophils, and fibrin. ${ }^{62}$ Increased platelet-neutrophil aggregates were also detected in investigations of the platelet transcriptome in COVID-19. ${ }^{53}$ These studies $^{35,53,61,62}$ suggest that neutrophils, platelets, and NETs may contribute to inflammatory lung injury and systemic thromboinflammation in COVID-19-associated ARDS. ${ }^{60}$ Nevertheless, participation and contributions of neutrophils to COVID-19 and its complications remain to be defined.

\section{VASCULOPATHY AND THROMBOINFLAMMATION ARE COMMON IN CLASSIC ACUTE RESPIRATORY DISTRESS SYNDROME AND CORONAVIRUS DISEASE 2019 LUNG INFECTION}

Vasculopathy is a central component of DAD in acute lung injury and classic ARDS of diverse causes. ${ }^{4,17-19,21}$ Endothelial dysfunction, with endothelial cells injured but largely intact by ultrastructural examination, contributes to increased alveolarcapillary permeability to protein and interstitial and alveolar edema in the exudative phase of DAD; alterations in the endothelial network can be dramatic in the later proliferative phase. ${ }^{18,19}$ Thrombosis of pulmonary vessels is a common feature of both the exudative and proliferative phases. In systematic studies of lung vessels by postmortem angiography and histology correlated with antemortem balloon arteriography, vascular occlusion was detected in as many as $95 \%$ of cases. By microscopic examination, platelet-fibrin thrombi were commonly observed in alveolar capillaries and arterioles in acute ARDS, and platelet-fibrin thrombi in microvessels and laminated fibrin clots in preacinar and intra-acinar arteries were detected across the time line spectrum. The pulmonary vascular bed was found to be extensively remodeled in later proliferative and fibrotic phases of ARDS in lungs from some patients. ${ }^{19}$ 
Vasculopathy is also a component of acute lung injury in COVID-19, and it is suggested that SARS-CoV-2 induces a distinct vascular endotype of ARDS. ${ }^{8}$ Pulmonary vascular injury was identified in 16 out of 23 early reports of acute lung injury in COVID$19 .{ }^{11}$ In a quantitative histologic study that also used micro-computed tomography, ultrastructural analysis, and molecular assays, lungs from decedents with COVID-19 respiratory failure or ARDS secondary to $\mathrm{H} 1 \mathrm{~N} 1$ influenza were equally likely to have thrombi in precapillary pulmonary arteries, but alveolar-capillary microthrombi were 9 times more prevalent in patients with COVID-19. Structurally deformed capillaries with evidence for intussusceptive angiogenesis were also significantly more frequent in the lungs of subjects with COVID-19 compared with those with influenza, although the number of patients in each group was small. ${ }^{15}$ In a different analysis, platelet and/ or fibrin microthrombi were identified in lungs from $84 \%$ of 68 patients with COVID-19, several whom had large vessel pulmonary thrombi and extrapulmonary thrombotic involvement. ${ }^{14}$ Macrovascular and microvascular thrombi have been reported in multiple additional studies of COVID-19 lungs. ${ }^{10,12,23-27,29,32,61-63}$

Endotheliitis (also termed endothelialitis) has been identified in lungs from patients with COVID-19 ${ }^{15,33}$ and SARS-CoV-2-infected rhesus macaques. ${ }^{45}$ The term implies endothelial injury and dysfunction with a component of perivascular inflammation, but there is not yet a consensus definition or rigorous determination of whether it is commonly present in COVID-19 pneumonia or represents a unique histologic subphenotype. Endothelial damage, swelling, and vacuolization shown by light and electron microscopy have been attributed to endothelial cell infection by SARS-CoV-2 via ACE2, but, in some studies, viral particles or antigen were detected in AECs but not in capillary endothelium. ${ }^{11,15,22,24,32,33,36,56}$ Perivascular leukocyte involvement has been reported as lymphocytic angiocentric inflammation ${ }^{15,45}$ and lymphocytic and myeloid cell accumulation in different patients, ${ }^{33}$ intravascular fibrin deposition with septal accumulation of neutrophils, ${ }^{34}$ and neutrophilic capillaritis. ${ }^{22,60}$ In 1 report, focal microthrombi were found, but no histologic evidence of endotheliitis. ${ }^{56}$ Perivascular inflammation did not reliably separate COVID-19 from other causes of DAD in a comparative analysis. ${ }^{29}$ Thus, there are many questions related to pulmonary endotheliitis in COVID-19 to be resolved. In addition, there are multiple fundamental issues to be explored. For example, it may be critical to know whether SARS-CoV-2-induced acute lung injury differentially affects recently described alveolar endothelial cell subtypes. ${ }^{64}$ Direct contributions of injured endothelial cells to other facets of alveolar damage and inflammation (see Fig. 1), and to viral containment versus spread, are also yet to be examined.

Thrombocytopenia, which is common and often profound in critically ill patients with classic ARDS, ${ }^{49}$ is generally mild in COVID-19, although it can be progressive in nonsurvivors. ${ }^{65}$ Platelets have dual roles as hemostatic and immune effector cells in the lung and other tissues, ${ }^{54}$ and can have thromboinflammatory activities in ARDS. ${ }^{49} \mathrm{Ag}$ gregates of platelets and platelet-fibrin thrombi have been commonly observed in microvessels in SARS-CoV-2-infected lungs, ${ }^{10,14,24-26,62,63}$ and molecular signatures associated with platelet activation, aggregation, and adhesion were detected in infected macaques. ${ }^{45}$ Altered platelet reactivity was detected in mild and severe COVID-19 infection. ${ }^{52,53,62}$ Platelet-neutrophil aggregates, a marker of platelet activation, ${ }^{54}$ were observed in COVID-19 lungs and blood. ${ }^{35,53,62}$ Platelets trigger NETosis, are effectors of pathologic clotting, ${ }^{49,60,65}$ and may contribute to NET formation and immunothrombosis in COVID-1961,62,66 (discussed earlier).

Megakaryocytes, the precursors of platelets, are present in normal and injured lungs and generate platelets in the pulmonary compartment (reviewed by Middleton and colleagues ${ }^{49}$ ). Megakaryocytes, including cells actively producing platelets, were detected in lung microvessels of decedents of COVID-19. ${ }^{10,26}$ Two autopsy series 
that included patients dying of COVID-19 or of classic ARDS found increased numbers of megakaryocytes in the pulmonary vasculature of subjects with COVID-19 infection, although only 1 comparison reached statistical significance. ${ }^{63,67}$ Megakaryocyte transcript signatures were increased in blood from patients ${ }^{68}$ and macaques ${ }^{45}$ infected with SARS-CoV-2. It is unknown whether megakaryocytes in the lungs of patients with COVID-19 have anti-SARS-CoV-2 activities that may influence COVID-19 infection, as with other viruses. ${ }^{69}$ In contrast, novel functions of megakaryocytes may contribute to acute lung injury or repair in COVID-19. ${ }^{49}$ Circulating megakaryocytes with a strong interferon-associated molecular signature were one of 3 cell types reported to be hallmarks of severe COVID-19 and were linked to inflammatory markers in plasma. ${ }^{68}$

Dysregulated systemic hemostasis may contribute to pulmonary vasculopathy in COVID-19. ${ }^{65}$ As markers, increase of plasma D-dimer concentration has been widely reported, and increased thrombin generation and relative impairment in fibrinolysis have been shown in severe COVID-19.6,65,70,71 Increased circulating plateletmonocyte aggregates were found in blood samples from patients with severe COVID-19 pneumonia, particularly those requiring mechanical ventilation. Platelets from these patients induced tissue factor expression by monocytes in vitro, ${ }^{52}$ suggesting a mechanism for pulmonary and systemic thromboinflammation. ${ }^{54}$

The causal links between deranged coagulation and macrothrombosis and microthrombosis in COVID-19 have not been elucidated, ${ }^{65}$ establishing key priorities for basic and clinical investigation. As an example, altered complement activation may link dysregulated inflammation and coagulation in COVID-19, including in patients with respiratory failure and systemic complications. ${ }^{34,65,66,72,73}$ The SARS-CoV-2 spike protein activates the alternate pathway of complement, ${ }^{74}$ suggesting a possible mechanism. SARS-CoV2 also induces complement and coagulation pathways in macaques. ${ }^{45}$ Lung and systemic manifestations were mitigated in C3-deficient mice infected with SARS-CoV-2. ${ }^{73}$

\section{PULMONARY COINFECTION IN CORONAVIRUS DISEASE 2019 PNEUMONIA: A COMMON PHENOTYPE}

Microbial coinfection is common in COVID-19-induced acute lung injury, based on autopsy series in which the frequency of suspected or documented bacterial or fungal coinfection, usually termed superimposed bronchopneumonia or superinfection, was $13 \%$ to $79 \% .{ }^{11,14,23-25,27,30,32,35,63}$ The histologic pattern has been described as dense accumulations of neutrophils in alveoli and airways, alveolar hemorrhage, and vascular congestion on a background of DAD with lymphocytic interstitial infiltrates. ${ }^{27}$ Special stains, microbial cultures, and molecular techniques have been used to confirm histologic findings and identify a variety of non-COVID-19 pathogens, ${ }^{14,27,30}$ although in some reports the diagnosis was made exclusively on the pattern of neutrophilic bronchopneumonia with DAD and, in others, coinfection was not detected. ${ }^{26}$ Frequent coinfection was also reported in a recent study of blood and airway myeloid leukocyte subsets in patients with severe COVID-19 lung involvement. ${ }^{55}$ For comparison, coinfection was present in $26 \%$ of 100 fatal cases of pandemic influenza A; DAD was a ubiquitous underlying histologic pattern. ${ }^{75}$ In contrast, coinfection was not frequently reported in the SARS and MERS pandemics. ${ }^{30}$

In classic ARDS, DAD, acute neutrophilic pneumonia caused by bacterial or fungal pathogens, and DAD together with acute neutrophilic pneumonia are each distinct histologic phenotypes that carry physiologic significance and have different clinical outcomes. ${ }^{21}$ This finding may also be true for COVID-19-associated ARDS, an issue that should be addressed in future clinical and experimental investigations. Application of metagenomic techniques will also be revealing. 


\section{ANIMAL MODELS: EXPERIMENTAL CORRELATES AND CONSISTENT HISTOPATHOLOGY}

Specific findings in primate models of SARS-CoV-2 lung infection were mentioned earlier. Although overt clinical signs do not develop short term, histopathology in cynomolgus macaques is consistent with that in human COVID-19 pneumonia (see Fig. 1). Foci of pulmonary consolidation were found in 2 of 4 animals (young and aged) 4 days after infection with SARS-CoV-2 isolated from a human patient. ${ }^{57}$ The major histologic changes included areas of acute lung injury with features of exudative and proliferative DAD, indicating that this pattern of response is a facet of the biology of SARS-CoV-2 lung infection that can develop rapidly and supporting the conclusion that DAD is not exclusively secondary to respiratory therapy measures. ${ }^{29}$ DAD histopathology was also observed in animals infected in parallel with MERS-CoV for comparison. SARS-CoV-2 antigen was detected in AEC I and AEC II in areas of DAD, a proximity that suggested that SARS-CoV-2 infection drives evolution of the DAD histologic pattern. ${ }^{57}$ Similarly, SARS-CoV-2 antigen and viral RNA were detected in focal areas of DAD in human COVID-19 pneumonia. ${ }^{14,32}$

Histologic changes of DAD also occur in rhesus macaques infected with SARSCoV-2. Hyaline membranes, alveolar edema, fibrin deposition, interstitial infiltrates, and AEC II hyperplasia were observed in multifocal areas of involvement 3 days after infection. ${ }^{59}$ In a second study, hyaline membranes were present together with interstitial and alveolar inflammatory infiltrates and edema 2 days after infection. ${ }^{58}$ In a study to examine age as a variable, histologic findings were generally similar but the specific components of interstitial inflammation and edema were more severe in aged rhesus macaques than in younger animals 7 days after SARS-CoV-2 infection. ${ }^{76}$ In cynomolgus macaques, age did not affect the histologic pattern at an early time point, but aged animals shed SARS-CoV-2 longer after infection. ${ }^{57}$

Additional animal models of COVID-19 have been developed, including experimental infection of rodents. ${ }^{40}$ Severe disease has been reported in some, depending on the animal species, genetic background, and conditions of viral challenge. ${ }^{40,77-79}$ To date, studies of mice have largely described alveolar interstitial infiltrates and edema, recapitulating some but not all of the histologic determinants of DAD (see Fig. 1). Acute lung injury with hyaline membranes and other DAD features were reported in 2 recently described murine models. ${ }^{77,79}$

Although the rapidly expanding repertoire of primate and small animal models will provide correlates of human COVID-19 and experimental infrastructure for mechanistic studies and for evaluation of therapeutics and vaccines, none yet developed is a faithful surrogate for human COVID-19-associated ARDS. ${ }^{40}$ As existing models are further refined and others developed for this purpose, lung histology will be a critical variable, as it has been in experimental acute lung injury of other causes. ${ }^{80} \mathrm{~A}$ recently reported model in standard laboratory mouse strains infected with a murine-adapted SARS-CoV-2 was interpreted to have features of ARDS based on assessment of acute lung injury and DAD by histologic scoring, ${ }^{77}$ although oxygenation and other key determinants of $\operatorname{ARDS}^{3,4,80}$ were not examined. The severity of injury was greater in BALB/c than in C57/BL6 mice.

\section{SUMMARY}

In January of 2020, nothing was known of the anatomic basis for respiratory failure caused by the novel SARS-CoV-2 virus. Since that time, examination of lung tissue from patients with COVID-19 has yielded a body of information with clinical and basic relevance, showing the unique value of analysis of pathologic anatomy in emerging 
and reemerging infectious diseases. ${ }^{11}$ The findings provide an initial foundation for understanding pathophysiologic features of COVID-19-associated $\operatorname{ARDS}^{1,2,5,6,16}$ and priorities for future investigations.

It is clear that SARS-CoV-2 infection can cause injury from the tracheobronchial epithelium to the pleura. ${ }^{10-13}$ Injury to the alveoli characterized by DAD is central to COVID-19 respiratory failure, consistent with the histopathology of severe infections caused by other respiratory viruses, including SARS-CoV, MERS-CoV, and influenza. ${ }^{11,12,17,75}$ Features of DAD were also prominent in lungs of patients reported in the original description of ARDS, several of whom were thought to have fatal viral pneumonia. ${ }^{81}$ Pulmonary vascular and microvascular involvement is common in COVID-19 pneumonia and may have unique features. DAD and its microvascular component are histologic counterparts for altered compliance, dead space, and oxygenation and for perfusion and other imaging abnormalities in patients with COVID-19-associated ARDS. ${ }^{6}$ Further study may provide additional useful insights, as it has in classic ARDS. ${ }^{19,21,41,42}$ Although the common histopathologic and cellular features of established COVID-19 acute lung injury are becoming clear, the early events in alveolar damage, the temporal progression to DAD, and the cellular and molecular mechanisms involved are obscure. Further definition of these features may have therapeutic significance. Current anatomic findings with clinical correlates suggest that pathways inherent to both the pathogen and the host contribute to COVID-19-associated ARDS. ${ }^{12,15,31,82,83}$

Findings from examination of COVID-19 lung tissue raise many additional questions and priorities for investigation, some of which are identified in this article, and potential controversies. The frequency, mechanisms, and physiologic significance of histologic patterns that vary from established $\mathrm{DAD}^{10,11,14,22,25,27,33-36}$ are open questions that need to be resolved. The specific issue of endotheliitis, which is currently based on a small number of diverse observations, needs further definition. The extent to which alveolar microvascular involvement is greater, or not different, compared with that of classic ARDS, ${ }^{15,29}$ potential SARS-CoV-2-specific mechanisms of alveolar vasculopathy, ${ }^{15,45,79}$ and whether COVID-19-associated ARDS is a distinct vascular endotype of $\mathrm{ARDS}^{8}$ each merit additional examination. Similarly, the specific contributions of neutrophils, NETs, platelets, and megakaryocytes to the pathogenesis of SARS-CoV-2 pneumonia and COVID-19-associated ARDS, in the broader context of activities of key immune effector cells, are unresolved issues with possible therapeutic relevance. Histopathologic analysis amplified by the tools of modern biology and molecular immunology, ${ }^{15,31}$ in parallel with rigorous animal models ${ }^{40}$ and reduced experimental approaches including isolated cell-based assays and organoid preparations, has the potential to provide needed answers and contribute to vetting of emerging hypotheses. ${ }^{84}$ Such insights may then refine understanding of the place of COVID-19 acute lung injury in the broader spectrum of ARDS. ${ }^{2-4,7-9}$

\section{CLINICS CARE POINTS}

- DAD has been consistently identified as the key histologic pattern of acute lung injury in SARS-CoV-2-associated pneumonia and ARDS.

- The histologic pattern of DAD in SARS-CoV-2-associated lung injury is not different from DAD in classic ARDS not associated with SARS-CoV-2 infection and COVID-19 pneumonia.

- Biological activities of injured alveolar cells and immune effector cells recruited to the alveoli in SARS-CoV-2-associated pneumonia and ARDS may contribute to unique physiologic responses and to outcomes in these syndromes. 
- Additional histologic patterns besides DAD have been identified in SARS-CoV-2-associated acute lung injury and may be the basis for heterogeneity and subphenotypes in COVID19-associated ARDS.

- COVID-19-associated ARDS is histologically similar to ARDS of other causes in the presence of DAD and perivascular inflammation; however, there is evidence of direct viral infection in the tracheobronchial epithelium and AECs.

- Deranged coagulation and presence of immunothrombi is a notable finding in COVID-19associated ARDS and has many potential drivers, including the presences of NETs, complement activation and increased platelet activation/aggregation, and increased monocyte tissue factor levels induced by platelet-monocyte aggregates.

- Lung tissue examined is largely from elderly patients who succumbed to the disease; therefore, distinguishing direct disease from SARS-CoV-2 infection or complex comorbid disease is challenging.

- Secondary bacterial and fungal pneumonias commonly occur in COVID-19-associated ARDS, although the clinical outcomes driven by the physiologic consequences of coinfection are not yet clear.

\section{ACKNOWLEDGMENTS}

The authors greatly appreciate the efforts and creative contributions of Kendra Richardson and Diana Lim in preparation of the article and figure. E.A. Middleton is supported by a research career development award (1 KO8 HL153953-02) from the National Heart, Lung, and Blood Institute and an award from the University of Utah Immunology, Inflammation, and Infectious Disease Program. G.A. Zimmerman was supported by awards from the National Institutes of Health (R37 HL044525, HL077671, HL130541, HD093826) while the work cited was done.

\section{DISCLOSURE}

The authors have nothing to disclose.

\section{REFERENCES}

1. Berlin DA, Gulik RM, Martinez FJ. Severe COVID-19. N Engl J Med 2020. https:// doi.org/10.1056/NEJMcp2009575.

2. Ware LB. Physiological and biological heterogeneity in COVID-19-associated acute respiratory distress syndrome. Lancet Respir Med 2020. https://doi.org/ 10.1016/S2213-2600(20)30369-6. S2213-2600(20)30369-6.

3. Thompson BT, Chambers RC, Liu KD. Acute respiratory distress syndrome. N Engl J Med 2017;377:562-72.

4. Matthay MA, Zemans RL, Zimmerman GA, et al. Acute respiratory distress syndrome. Nat Rev 2019;5:18.

5. Ferrando C, Suarez-Sipmann F, Mellado-Artigas R, et al. Clinical features, ventilatory management, and outcome of ARDS caused by COVID-19 are similar to other causes of ARDS. Intensive Care Med 2020. https://doi.org/10.1007/ s00134-020-06192-2.

6. Grasselli G, Tonetti T, Protti A, et al. Pathophysiology of COVID-19-associated acute respiratory distress syndrome: a multicentre prospective observational study. Lancet Respir Med 2020;8(12):1201-8.

7. Sinha P, Calfee CS, Cherian S, et al. Prevalence of phenotypes of acute respiratory distress syndrome in critically ill patients with COVID-19: a prospective 
observational study. Lancet Respir Med 2020. https://doi.org/10.1016/S22132600(20)30366-0. S2213-2600(20)30366-0.

8. Mangalmurti NS, Reilly JP, Cines DB, et al. COVID-19-associated acute respiratory distress syndrome clarified: a vascular endotype? Am J Respir Crit Care Med 2020;202(5):750-3.

9. Thompson BT, Matthay MA. The Berlin Definition of ARDS versus pathological evidence of diffuse alveolar damage. Am J Respir Crit Care Med 2013;187(7): $675-7$.

10. Buja LM, Wolf DA, Zhao B, et al. The emerging spectrum of cardiopulmonary pathology of the coronavirus disease 2019 (COVID-19): report of 3 autopsies from Houston, Texas, and review of autopsy findings from other United States cities. Cardiovasc Pathol 2020;48:107233.

11. Calabrese F, Pezzuto F, Fortarezza F, et al. Pulmonary pathology and COVID-19: lessons from autopsy. The experience of European Pulmonary Pathologists. Virchows Arch 2020;477(3):359-72.

12. Mohanty SK, Satapathy A, Naidu MM, et al. Severe acute respiratory syndrome coronavirus-2 (SARS-CoV-2) and coronavirus disease 19 (COVID-19) - anatomic pathology perspective on current knowledge. Diagn Pathol 2020;15:103.

13. Vasquez-Bonilla W, Orozco R, Argueta V, et al. A review of the main histopathological findings in coronavirus disease 2019. Hum Pathol 2020;105:74-83.

14. Borczuk AC, Salvatore SP, Seshan SV, et al. COVID-19 pulmonary pathology: a multi-institutional autopsy cohort from Italy and New York City. Mod Pathol 2020;33(11):2156-68.

15. Ackermann M, Verleden SE, Kuehnel M, et al. Pulmonary vascular endothelialitis, thrombosis, and angiogenesis in COVID-19. N Engl J Med 2020;383:120-8.

16. Wiersinga WJ, Rhodes A, Cheng AC, et al. Pathophysiology, transmission, diagnosis, and treatment of Coronavirus disease 2019 (COVID-19). JAMA 2020; 324(8):782-93.

17. Katzenstein AL, Bloor CM, Leibow AA. Diffuse alveolar damage-the role of oxygen, shock, and related factors. A review. Am J Pathol 1976;85(1):209-28.

18. Bachofen M, Weibel ER. Structural alterations of lung parenchyma in the adult respiratory distress syndrome. Clin Chest Med 1982;3(1):35-56.

19. Tomashefski JF Jr. Pulmonary pathology of acute respiratory distress syndrome. Clin Chest Med 2000;21(3):435-66.

20. Zemans RL, Matthay MA. What drives neutrophils to the alveoli in ARDS? Thorax 2017;72(1):1-3.

21. Cardinal-Fernández P, Lorente JA, Ballén-Barragán A, et al. Acute respiratory distress syndrome and diffuse alveolar damage. New insights on a complex relationship. Ann Am Thorac Soc 2017;14(6):844-50.

22. Bössmüller H, Traxier $S$, Bitzer $M$, et al. The evolution of pulmonary pathology in fatal COVID-19 disease: an autopsy study with clinical correlation. Virchows Arch 2020;477(3):349-57.

23. Bussani R, Schneider E, Zentilin L, et al. Persistence of viral RNA, pneumocyte syncytia and thrombosis are hallmarks of advanced COVID-19 pathology. EBioMedicine 2020;61:103104.

24. Carsana L, Sonzogni A, Nasr A, et al. Pulmonary post-mortem findings in a series of COVID-19 cases from northern Italy: a two-centre descriptive study. Lancet Infect Dis 2020;20(10):1135-40.

25. De Michele S, Sun Y, Yilaz MM, et al. Forty postmortem examinations in COVID-19 patients. Am J Clin Pathol 2020;154(6):748-60. 
26. Fox SE, Akmatbekov A, Harbert JL, et al. Pulmonary and cardiac pathology in African American patients with COVID-19: an autopsy series from New Orleans. Lancet Respir Med 2020;8(7):681-6.

27. Grosse C, Grosse A, Salzer HJF, et al. Analysis of cardiopulmonary findings in COVID-19 fatalities: high incidence of pulmonary artery thrombi and acute suppurative bronchopneumonia. Cardiovasc Pathol 2020;49:107263.

28. Hanley B, Naresh KN, Roufosse C, et al. Histopathological findings and viral tropism in UK patients with severe fatal COVID-19: a post-mortem study. Lancet Microbe 2020;1(6):e245-53.

29. Konopka KE, Nguyen T, Jentzen JM, et al. Diffuse alveolar damage (DAD) resulting from coronavirus disease 2019 infection is morphologically indistinguishable from other causes of DAD. Histopathology 2020;77(4):570-8.

30. Martines RB, Ritter JM, Matkovic E, et al. Pathology and pathogenesis of SARSCoV-2 associated with fatal Coronavirus Disease, United States. Emerg Infect Dis 2020;26(9):2005-15.

31. Nienhold R, Ciani Y, Koelzer VH, et al. Two distinct immunopathological profiles in autopsy lungs of COVID-19. Nat Commun 2020;11(1):5086.

32. Schaefer IM, Padera RF, Solomon IH, et al. In situ detection of SARS-CoV-2 in lungs and airways of patients with COVID-19. Mod Pathol 2020;1-11. https:// doi.org/10.1038/s41379-020-0595-Z.

33. Varga Z, Flammer AJ, Steiger $P$, et al. Endothelial cell infection and endotheliitis in COVID-19. Lancet 2020;395(10234):1417-8.

34. Magro C, Mulvey JJ, Berlin D, et al. Complement associated microvascular injury and thrombosis in the pathogenesis of severe COVID-19 infection: a report of five cases. Transl Res 2020;220:1-13.

35. Schurink B, Roos E, Radonic T, et al. Viral presence and immunopathology in patients with lethal COVID-19: a prospective autopsy cohort study. Lancet Microbe 2020;1(7):e290-9.

36. Copin MC, Parmentier E, Duburcq T, et al. Time to consider histologic pattern of lung injury to treat critically ill patients with COVID-19 infection. Intensive Care Med 2020;46(6):1124-6.

37. Pernazza A, Mancini M, Rullo E, et al. Early histologic findings of pulmonary SARS-CoV-2 infection detected in a surgical specimen. Virchows Arch 2020; 477(5):743-8.

38. Tian S, Hu W, Niu L, et al. Pulmonary pathology of early-phase 2019 novel Coronavirus (COVID-19) pneumonia in two patients with lung cancer. J Thorac Oncol 2020;15(5):700-4.

39. Zeng Z, Xu L, Xie XY, et al. Pulmonary pathology of early-phase COVID-19 pneumonia in a patient with a benign lung lesion. Histopathology 2020;77(5):823-31.

40. Muñoz-Fontela C, Dowling WE, Funnell SGP, et al. Animal models for COVID-19. Nature 2020;586(7830):509-15.

41. Thille AW, Esteban A, Fernández-Segoviano P, et al. Chronology of histological lesions in acute respiratory distress syndrome with diffuse alveolar damage: a prospective cohort study of clinical autopsies. Lancet Respir Med 2013;1(5): 395-401.

42. Thille AW, Esteban A, Fernández-Segoviano P, et al. Comparison of the Berlin definition for acute respiratory distress syndrome with autopsy. Am J Respir Crit Care Med 2013;187(7):761-7.

43. Cardinal-Fernández P, Bajwa EK, Dominguez-Calvo A, et al. The presence of diffuse alveolar damage on open lung biopsy is associated with mortality in 
patients with acute respiratory distress syndrome: a systematic review and metaanalysis. Chest 2016;149(5):1155-64.

44. Merad M, Martin JC. Pathological inflammation in patients with COVID-19: a key role for monocytes and macrophages. Nat Rev Immunol 2020;1-8. https://doi. org/10.1038/s41577-020-0331-4.

45. Aid M, Busman-Sahay K, Vidal SJ, et al. Vascular disease and thrombosis in SARS-CoV-2-infected rhesus macaques. Cell 2020;183(5):1354-66.

46. Giani M, Seminati D, Lucchini A, et al. Exuberant plasmocytosis in bronchoalveolar lavage specimen of the first patient requiring extracorporeal membrane oxygenation for SARS-CoV-2 in Europe. J Thorac Oncol 2020;15(5):e65-6.

47. Liao M, Liu Y, Yuan J, et al. Single-cell landscape of bronchoalveolar immune cells in patients with COVID-19. Nat Med 2020;26(6):842-4.

48. Voiriot G, Fajac A, Lopinto J, et al. Bronchoalveolar lavage findings in severe COVID-19 pneumonia. Intern Emerg Med 2020;15(7):1333-4.

49. Middleton EA, Rondina MT, Schwertz HJ, et al. Amicus or adversary revisited: platelets in acute lung injury and acute respiratory distress syndrome. Am J Respir Cell Mol Biol 2018;59(1):18-35.

50. Song JW, Zhang C, Fan X, et al. Immunological and inflammatory profiles in mild and severe cases of COVID-19. Nat Commun 2020;11(1):3410.

51. Boyd DF, Allen EK, Randolph AG, et al. Exuberant fibroblast activity compromises lung function via ADAMTS4. Nature 2020;587:466-71.

52. Hottz ED, Azevedo-Quintanilha IG, Palhinha L, et al. Platelet activation and platelet-monocyte aggregate formation trigger tissue factor expression in patients with severe COVID-19. Blood 2020;136(11):1330-41.

53. Manne BK, Denorme F, Middleton EA, et al. Platelet gene expression and function in patients with COVID-19. Blood 2020;136:1317-29.

54. Middleton EA, Weyrich AS, Zimmerman GA. Platelets in pulmonary immune responses and inflammatory lung diseases. Physiol Rev 2016;96(4):1211-59.

55. Sanchez-Cerrillo I, Landete P, Aldave B, et al. COVID-19 severity associates with pulmonary redistribution of $\mathrm{CD}_{1 \mathrm{C}}+\mathrm{DCs}$ and inflammatory transitional and nonclassical monocytes. J Clin Invest 2020;130(12):6290-300.

56. Bradley BT, Maioli H, Johnston R, et al. Histopathology and ultrastructural findings of fatal COVID-19 infections in Washington State: a case series. Lancet 2020;396(10247):320-32.

57. Rockx B, Kuiken T, Herfst S, et al. Comparative pathogenesis of COVID-19, MERS, and SARS in a nonhuman primate model. Science 2020;368(6494): 1012-5.

58. Chandrashekar A, Liu J, Martinot AJ, et al. SARS-CoV-2 infection protects against rechallenge in rhesus macaques. Science 2020;369(6505):812-7.

59. Munster VJ, Feldmann F, Williamson BN. Respiratory disease in rhesus macaques inoculated with SARS-CoV-2. Nature 2020;585(7824):268-72.

60. Barnes BJ, Adrover JM, Baxter-Stoltzfus A, et al. Targeting potential drivers of COVID-19: neutrophil extracellular traps. J Exp Med 2020;217(6):e20200652.

61. Middleton EA, He XY, Denorme F, et al. Neutrophil extracellular traps contribute to immunothrombosis in COVID-19 acute respiratory distress syndrome. Blood 2020;136(10):1169-79.

62. Nicolai L, Leunig A, Brambs S, et al. Immunothrombotic dysregulation in COVID19 pneumonia is associated with respiratory failure and coagulopathy. Circulation 2020;142(12):1176-89. 
63. Rapkiewicz AV, Mai X, Carsons SE, et al. Megakaryocytes and platelet-fibrin thrombi characterize multi-organ thrombosis at autopsy in COVID-19: a case series. EClinicalMedicine 2020;24:100434.

64. Gillich A, Zhang F, Farmer CG, et al. Capillary cell-type specialization in the alveolus. Nature 2020;586(7831):785-9.

65. Mackman N, Antoniak S, Wolberg AS, et al. Coagulation abnormalities and thrombosis in patients infected with SARS-CoV-2 and other pandemic viruses. Arterioscler Thromb Vasc Biol 2020;40(9):2033-44.

66. Skendros P, Mitsios A, Chrysanthopoulou A, et al. Complement and tissue factorenriched neutrophil extracellular traps are key drivers in COVID-19 immunothrombosis. J Clin Invest 2020;130(11):6151-7.

67. Valdivia-Mazeyra M, Salas C, Nieves-Alonso J, et al. Increased number of pulmonary megakaryocytes in COVID-19 patients with diffuse alveolar damage: an autopsy study with clinical correlation and review of the literature. Virchows Arch 2020;1-10. https://doi.org/10.1007/s00428-020-02926-1.

68. Bernardes JP, Mishra N, Tran F, et al. Longitudinal multi-omics analyses identify responses of megakaryocytes, erythroid cell, and plasmablasts as hallmarks of severe COVID-19. Immunity 2020;53(6):1296-314.

69. Campbell RA, Schwertz H, Hottz ED, et al. Human megakaryocytes possess intrinsic antiviral immunity through regulated induction of IFITM3. Blood 2019; 133(19):2013-26.

70. Bouck EG, Denorme F, Holle LA, et al. COVID-19 and sepsis are associated with different abnormalities in plasma procoagulant and fibrinolytic activity. Arterioscler Thromb Vasc Biol 2020;41(1):401-14.

71. Ranucci M, Sitzia C, Baryshnikova E, et al. COVID-19-associated coagulopathy: biomarkers of thrombin generation and fibrinolysis leading the outcome. $J$ Clin Med 2020;9(11):3487.

72. Cugno M, Meroni PL, Gualtierotti R, et al. Complement activation in patients with COVID-19: a novel therapeutic target. J Allergy Clin Immunol 2020;146(1):215-7.

73. Gralinski LE, Sheahan TP, Morrison TE, et al. Complement activation contributes to severe acute respiratory syndrome coronavirus pathogenesis. mBio 2018;9(5): e01753-18.

74. Yu J, Yuan $\mathrm{X}$, Chen $\mathrm{H}$, et al. Direct activation of the alternative complement pathway by SARS-CoV-2 spike proteins is blocked by factor D inhibition. Blood 2020;136(18):2080-9.

75. Shieh WJ, Blau DM, Denison AM, et al. 2009 pandemic influenza A (H1N1): pathology and pathogenesis of 100 fatal cases in the United States. Am J Pathol 2010;177(1):166-75.

76. Yu P, Qi F, Xu Y, et al. Age-related rhesus macaque models of COVID-19. Anim Model Exp Med 2020;3(1):93-7.

77. Leist SR, Dinnon KH 3rd, Schäfer A, et al. A mouse-adapted SARS-CoV-2 induces acute lung injury and mortality in standard laboratory mice. Cell 2020; 183(4):1070-85.e12.

78. Tostanoski LH, Wegmann F, Martinot AJ, et al. Ad26 vaccine protects against SARS-CoV-2 severe clinical disease in hamsters. Nat Med 2020;26(11): 1694-700.

79. Zheng J, Wong LYR, Li K, et al. COVID-19 treatments and pathogenesis including anosmia in K18-hACE2 mice. Nature 2020. https://doi.org/10.1038/s41586-0202943-z. 
80. Matute-Bello G, Downey G, Moore BB, et al. An official American Thoracic Society workshop report: features and measurements of experimental acute lung injury in animals. Am J Respir Cell Mol Biol 2011;44(5):725-38.

81. Ashbaugh DG, Bigelow DB, Petty TL, et al. Acute respiratory distress in adults. Lancet 1967;2(7511):319-23.

82. Hue S, Beldi-Ferchiou A, Bendib I, et al. Uncontrolled innate and impaired adaptive immune responses in patients with COVID-19 acute respiratory distress syndrome. Am J Respir Crit Care Med 2020;202(11):1509-19.

83. Matthay MA, Leligdowicz A, Liu KD. Biological Mechanisms of COVID-19 acute respiratory distress syndrome. Am J Respir Crit Care Med 2020;202(11): 1489-91.

84. Grant RA, Morales-Nebreda L, Markov NS, et al. Circuits between infected macrophages and T cells in SARS-CoV-2 pneumonia. Nature 2021. https://doi.org/10. 1038/s41586-020-03148-w. 